\title{
Insulin-like growth factor 1 receptor-mediated cell survival in hypoxia depends on the promotion of autophagy via suppression of the PI3K/Akt/mTOR signaling pathway
}

\author{
QI LIU ${ }^{1,2}$, JING-ZHI GUAN ${ }^{3}$, YONG SUN ${ }^{1,2}$, ZIYU LE $^{2,4}$, PING ZHANG $^{1,2}$, DONG YU $^{5}$ and YONG LIU ${ }^{1,2}$ \\ ${ }^{1}$ Cancer Research Institute, Fudan University Shanghai Cancer Center; ${ }^{2}$ Department of Oncology, \\ Shanghai Medical College, Fudan University, Shanghai 200032; ${ }^{3}$ Department of Oncology, \\ The People's Liberation Army No. 309 Hospital, Beijing 100193; ${ }^{4}$ Department of Radiation Oncology, \\ Fudan University Shanghai Cancer Center, Fudan University, Shanghai 200032; ${ }^{5}$ School of Radiological \\ Medicine and Protection, Medical College of Soochow University, Soochow University, Suzhou, Jiangsu 215123, P.R. China
}

Received December 2, 2015; Accepted December 15, 2016

DOI: $10.3892 / \mathrm{mmr} .2017 .6265$

\begin{abstract}
Hypoxia is widely accepted as a fundamental biological phenomenon, which is strongly associated with tissue damage and cell viability under stress conditions. Insulin-like growth factor-1 (IGF-1) is known to protect tissues from multiple types of damage, and protect cells from apoptosis. Hypoxia is a regulatory factor of the IGF system, however the role of the IGF-1 receptor (IGF-1R) in hypoxia-induced apoptosis remains unclear. The present study investigated the potential mechanisms associated with IGF-1R-associated apoptosis under hypoxic conditions. Mouse embryonic fibroblasts exhibiting disruption or overexpression of IGF-1R ( $\mathrm{R}^{-}$cells and $\mathrm{R}^{+}$cells) were used to examine the level of apoptosis, autophagy, and production of reactive oxygen species (ROS). The autophagy inhibitor 3-methyladenine was used to assess the effect of autophagy on ROS production and apoptosis under hypoxic conditions. A potential downstream
\end{abstract}

Correspondence to: Professor Yong Liu, Cancer Research Institute, Fudan University Shanghai Cancer Center, 270 Dong An Road, Shanghai 200032, P.R. China

E-mail: drliuyrt@163.com

Professor Dong Yu, School of Radiological Medicine and Protection, Medical College of Soochow University, Soochow University, 199 Ren Ai Road, Suzhou Industrial Park, Suzhou, Jiangsu 215123, P.R. China

E-mail: yudong@suda.edu.cn

Abbreviations: Akt, threonine protein kinase B; DCHF-DA, dichloro-dihydro-fluorescein diacetate; H2DCFDA, 2', 7'-dichlorodihydrofluorescein diacetate; IGF-1R, insulin-like growth factor-1 receptor; 3MA, 3-methyladenine; mTOR, mammalian target of rapamycin; PI3K, phosphatidylinositol 3-kinase; ROS, reactive oxygen species

Key words: insulin-like growth factor 1 receptor, reactive oxygen species, hypoxia, autophagy, apoptosis signaling pathway involving phosphatidylinositol 3-kinase $(\mathrm{PI} 3 \mathrm{~K}) /$ threonine protein kinase $\mathrm{B}(\mathrm{Akt}) /$ mammalian target of rapamycin (mTOR) was identifiedby western blot analysis. The results demonstrated that hypoxia induced apoptosis, increased ROS production, and promoted autophagy in a time-dependent manner relative to that observed under normoxia. $\mathrm{R}^{+}$cells exhibited a lower percentage of apoptotic cells, lower ROS production, and higher levels of autophagy when compared to that of R- cells. In addition, inhibition of autophagy led to increased ROS production and a higher percentage of apoptotic cells in the two cell types. Furthermore, IGF-1R is related with PI3K/Akt/mTOR signaling pathway and enhanced autophagy-associated protein expression, which was verified following treatment with the PI3K inhibitor LY294002. These results indicated that IGF-1R may increase cell viability under hypoxic conditions by promoting autophagy and scavenging ROS production, which is closed with PI3K/Akt/mTOR signaling pathway.

\section{Introduction}

Hypoxia is a fundamental biological phenomenon that is strongly associated with tissue damage and cell viability under stress conditions. It is widely accepted that hypoxic foci are present in the microenvironment during ischemic injuries, including neurological (1), intestinal (2), myocardial (3) and liver (4) damage. These affect mitochondrial respiratory chain functions, mitochondrial enzymes and adenosine triphosphate synthesis (5). In addition, hypoxia develops in normal tissues following radiation exposure and is associated with increased inflammatory corpuscle accumulation and activation, oxidative stress, and profibrogenic cytokine activity, thus contributing to radiation-induced normal tissue injury $(6,7)$. Reactive oxygen species (ROS)-dependent apoptosis via attenuation of mitochondrial function and signaling pathways, has been demonstrated to be a major cause of hypoxia-associated tissue injury (8-11).

Insulin-like growth factor-1 (IGF-1) functions to promote a survival and proliferation in specific tissues by initiating 
signaling cascades following binding to extracellular IGF-1 receptor (IGF-1R), which results in IGF-1 activation and phosphorylation (12). IGF-1R is a member of the tyrosine kinase receptor superfamily, which is involved in the regulation of cell proliferation, differentiation, and survival (12). A previous study demonstrated that IGF-1R is involved in apoptosis induction through the reduction of mitochondrial dysfunction (13). The protective mechanisms associated with IGF-1R involve preservation of the mitochondrial membrane potential and reduction of caspase-3 activity (13). A previous study indicated that IGF-1R in the endothelium maintains the endothelial barrier by stabilizing the vascular endothelial (VE)-protein tyrosine phosphatase/VE-cadherin complex (14). Furthermore, decreased IGF-1R expression impairs endothelial function and increases renal fibrosis, which is associated with kidney disease (14). Similarly, IGF-1R is essential in mediating IGF activity during neuronal cell development. IGF-1R in neuronal cells is critically important for their survival following hypoxic/ischemic (H/I) injury (1). IGF-mediated upregulation of the neuronal cellular inhibitor of apoptosis-1 and X-linked inhibitor of apoptosis protein, contribute to IGF/IGF-1R protection against neuronal apoptosis following H/I injury (1). In addition, IGF/IGF-1R have been demonstrated to protect against intestinal and cardiomyocyte ischemic-reperfusion (I/R) injury $(2,3)$.

Hypoxia is one of the factors involved in the regulation of the IGF system $(15,16)$. IGF-1R expression and concentration are altered when cells, such as human hepatocytes and growth neuronal cones, are exposed to hypoxic conditions $(15,16)$. Additionally, IGF-1/IGF-1R protect cultured human cells against a variety of injuries, such as oxidative stress and hypoxia, through the activation of associated proteins including nuclear factor- $\kappa \mathrm{B}$ and cyclic adenosine monophosphate-response element binding protein (17-19). However, the mechanisms associated with its anti-apoptotic effects remains unknown. The present study demonstrates an association between IGF-1R and the phosphatidylinositol 3-kinase (PI3K)/threonine protein kinase B (Akt)/mammalian target of rapamycin mTOR signaling pathway and autophagy. In addition, these results provide evidence supporting the protective role of IGF-1R against oxidative stress under hypoxic conditions.

\section{Materials and methods}

Cell lines and reagents. $\mathrm{R}^{-}$and $\mathrm{R}^{+}$cells were a gift from Dr. Yu Dong (Soochow University, Suzhou, China). R- cells were fibroblast cell lines derived from mouse embryos with targeted disruption of the IGF-1R genes (20). $\mathrm{R}^{+}$cells were derived from $\mathrm{R}^{-}$ cells following co-transfection with a human IGF-1R expression plasmid and a pLHL4 plasmid carrying the hygromycin resistance gene (20,21). All cells were cultured in Dulbecco's modified Eagle's medium (Biowest, Nuaillé, France) supplemented with 1\% penicillin/streptomycin (P0781; Sigma-Aldrich; Merck Millipore, Darmstadt, Germany) and 10\% fetal bovine serum (Biowest) in a free-gas exchange chamber with atmospheric air at $37^{\circ} \mathrm{C}$. Hypoxia treatment $(24$ or $48 \mathrm{~h}$ ) was performed in a tri-gas incubator (YCP-50S; Huaxi Electronic Technology Co., Ltd., Hunan, China) at $37^{\circ} \mathrm{C}, 5 \% \mathrm{CO}_{2}, 93 \% \mathrm{~N}_{2}$ and $2 \% \mathrm{O}_{2}$. The PI3K inhibitor LY294002 (Sigma-Aldrich; Merck Millipore) was used for $24 \mathrm{~h}$ at $10 \mu \mathrm{M}$. The autophagy inhibitor 3-methyladenine (3MA; M9281-100MG; Sigma-Aldrich; Merck Millipore) was used at a concentration of $5 \mu \mathrm{M}$ for $24 \mathrm{~h}$.

$R N A$ isolation and quantitative polymerase chain reaction ( $q P C R)$. R- and R+ cells were grown to a number of $10^{7}$ and then digested with trypsin. The total RNA was isolated using a High Pure RNA Isolation kit (RNAprep Pure; Tiangen Biotech Co., Ltd., Beijing, China) and to eliminate genomic DNA contamination with with DNase I (Tiangen Biotech Co., Ltd.) according to the manufacturer's instructions. Total RNA (500 ng) was used as a template for reverse transcription reactions using the PrimeScript RT Reagent kit (Perfect Real Time; Takara Biotechnology Co., Ltd., Dalian, China), followed by $\mathrm{qPCR}$ analysis. The PCR procedures were performed as follows: Predenaturing at $95^{\circ} \mathrm{C}$ for $5 \mathrm{~min}$ followed by 40 cycles of amplifications by denaturing at $95^{\circ} \mathrm{C}$ for $15 \mathrm{sec}$, annealing for $30 \mathrm{sec}$ at $64^{\circ} \mathrm{C}$ for IGF-1R and $60^{\circ} \mathrm{C}$ for GAPDH, then extension at $72^{\circ} \mathrm{C}$ for $30 \mathrm{sec}$, followed by a final extension step at $72^{\circ} \mathrm{C}$ for $10 \mathrm{~min}$. Relative expression of target genes was analyzed by the $\Delta \Delta \mathrm{Cq}$ method (22). GAPDH was used as an internal control. The following primers were used for quantitative PCR: IGF-1R sense, 5'-TACAACTACGCCCTGGTCATC-3', and antisense, 5'-CTTCTCACACATCGGCTTCTC-3'; GAPDH, sense, 5'-TGAAGGTCGGTGTGAACGGATTTGG-3', and antisense, 5'-ACGACATACTCAGCACCGGCCTCAC-3'. IGF-1R expression was evaluated using the LightCycler 480 SYBR Green I Master kit (Roche Diagnostics, Basel, Switzerland).

Western blotting. Cells were grown to a number of $10^{7}$ and were lysed with radioimmunoprecipitation assay lysis buffer (Roche Diagnostics) and total protein was quantified using the Pierce BCA Protein assay kit (Thermo Fisher Scientific, Inc., Waltham, MA, USA) according to the manufacturer's instructions. Equal quantities of protein $(20 \mu \mathrm{g})$ were separated using SDS-PAGE on a $10 \%$ (w/v) polyacrylamide gel, then were electrotransferred onto a BioTrace NC Membrane (Pall Life Sciences, Port Washington, NY, USA). Blots on NC membrane were blocked for $1 \mathrm{~h}$ with blocking buffer consisting of $5 \%(\mathrm{w} / \mathrm{v})$ bovine serum albumin (Biowest) and $0.1 \%$ (v/v) Tween 20 (Sigma-Aldrich; Merck Millipore) in phosphate-buffered saline (PBS; HyClone; GEHealthcare Life Sciences, Logan, UT, USA). The antibodies used for western blotting were as follows: PI3K-110 (\#4252S; 1:1,000; Cell Signaling Technology, Inc., Danvers, MA, USA), phosphorylated (p)-Akt/Akt (\#14293/\#12178; 1:1,000; Cell Signaling Technology, Inc.), p-mTOR/mTOR (\#5536S/\#2983S; 1:1,000; Cell Signaling Technology, Inc.), and autophagy marker light chain 3 (M186-3; LC3; 1:1,000; Medical and Biological Laboratories Co., Ltd., Nagoya, Japan) and $\beta$-actin (\#12620; 1:1,000; Cell Signaling Technology, Inc.). Primary antibodies were incubated overnight at $4^{\circ} \mathrm{C}$, followed by incubation with horseradish peroxidase-conjugated secondary anti-rabbit (AP187R; 1:10,000; EMD Millipore, Billerica, MA, USA) or anti-mouse IgG antibodies (GTX26709; 1:10,000; GeneTex, Inc., Irvine, CA, USA) for $1 \mathrm{~h}$ at $37^{\circ} \mathrm{C}$. Protein bands were detected using the ECL Blotting Detection Reagent (Thermo Fisher Scientific, Inc.), and images were quantified using the Chemioscope Mini system (Bioshine, Shanghai, China). 
Autophagy detection. Cells were grown to a number of $10^{7}$, digested and washed twice with PBS ( $\mathrm{pH}$ 7.4; HyClone; Thermo Fisher Scientific, Inc.), centrifuged at $175 \times \mathrm{g}$ for 5 min at $37^{\circ} \mathrm{C}$, and resuspended in a PBS. The autophagosomes were marked and stained using the Cyto-ID Autophagy Detection kit (Enzo Biochem, Inc., New York, NY, USA) and photographed using a fluorescence microscope (Nikon Corporation, Tokyo, Japan). Fluorescence intensity was detected by flow cytometry (FC 500 MPL; Beckman Coulter, Inc., Brea, CA, USA).

Apoptosis detection. Cells were grown to a number of $10^{7}$, digested with trypsin and washed twice with PBS (pH 7.4; HyClone; GE Healthcare Life Sciences), centrifuged at 112 x $g$ for $5 \mathrm{~min}$ at $37^{\circ} \mathrm{C}$, and resuspended in PBS. Cells were stained using the Annexin V-Fluorescein Isothiocyanate Apoptosis Detection kit (BD Biosciences, Franklin Lakes, NJ, USA), and propidium iodide (BD Biosciences) according to the manufacturer's instructions. The apoptosis was analyzed using flow cytometry (FC 500 MPL; Beckman Coulter, Inc.).

ROS-generation assay. The intracellular ROS production levels were determined using a spectrofluorimetric method, H2DCFDA (Beyotime Institute of Biotechnology, Haimen, China) assay. $\mathrm{R}^{-}$and $\mathrm{R}^{+}$cells were grown to a number of $10^{7}$ and exposed to hypoxic conditions for 24 and $48 \mathrm{~h}$. Cells were then incubated with DCHF-DA $(20 \mathrm{mM})$ for $20 \mathrm{~min}$ at $37^{\circ} \mathrm{C}$ in a dark room. Subsequently, the cells were harvested in a trypsin-EDTA acid solution. Cell suspensions were centrifuged at $175 \mathrm{x} g$ for $5 \mathrm{~min}$ at $37^{\circ} \mathrm{C}$, then the supernatant was removed. The intensity of DCHF-DA fluorescence was measured and calculated by flow cytometry analysis (FC 500 MPL; Beckman Coulter, Inc.).

Statistical analysis. All experiments were performed in triplicate. Data were expressed as the mean \pm standard deviation. Statistical analysis was performed using the paired Student's $t$-test with SPSS software, version 22.0 (IBM SPSS, Armonk, NY, USA) considering the variances unequal. $\mathrm{P}<0.05$ was considered to indicate a statistically significant difference.

\section{Results}

Effect of IGF-1R on hypoxia-induced apoptosis. $\mathrm{R}^{+}$cells exhibited significantly higher levels of mRNA expression compared with that of $\mathrm{R}^{-}$cells (Fig. 1). Following treatment of hypoxia for 24 or $48 \mathrm{~h}, \mathrm{R}^{+}$cells and $\mathrm{R}^{-}$cells all exhibited increased apoptotic ratios compared with that of normoxic treatment, respectively. Further analysis showed hypoxia induced more apoptosis in $\mathrm{R}^{-}$cells compared with that of $\mathrm{R}^{+}$ cells under hypoxic conditions (Fig. 2).

Effects of IGF-1R on hypoxia-induced ROS production. To investigate whether IGF-1R alters cellular ROS levels under hypoxic conditions, ROS production in $\mathrm{R}^{-}$and $\mathrm{R}^{+}$cells under hypoxic conditions for 24 and $48 \mathrm{~h}$ was examined. As shown in Fig. 3, ROS production levels in $\mathrm{R}^{-}$and $\mathrm{R}^{+}$cells was increased significantly under hypoxic conditions compared with those under normoxic conditions. Additionally, $\mathrm{R}^{+}$cells produced significantly lower ROS levels under hypoxic conditions.

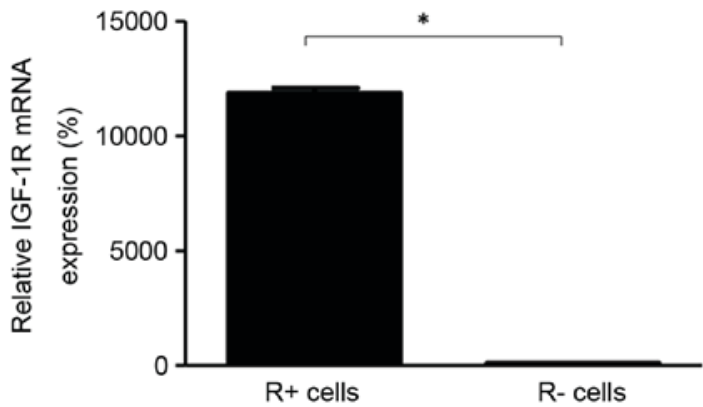

Figure 1. Expression of IGF-1R mRNA in $\mathrm{R}^{-}$and $\mathrm{R}^{+}$cells. Transfection efficiency was assessed by reverse transcription-quantitative polymerase chain reaction, and the relative IGF-1R mRNA expression levels $\left(\mathrm{R}^{+} / \mathrm{R}^{-}\right)$in $\mathrm{R}^{+}$and $\mathrm{R}^{-}$cells are shown. ${ }^{*} \mathrm{P}<0.05$. IGF-1R, insulin-like growth factor 1 receptor; $\mathrm{R}^{+}$ cells, mouse embryonic fibroblasts overexpressing IGF-1R; R' cells, mouse embryonic fibroblasts with targeted disruption of IGF-1R.

Under hypoxic conditions, the ROS levels were significantly higher in $\mathrm{R}^{-}$cells than in $\mathrm{R}^{+}$cells (Fig. 3 ).

Effects of IGF-1R on hypoxia-induced autophagy. As presented in Fig. 4, hypoxia increased the presence of autophagosome in all $\mathrm{R}^{-}$and $\mathrm{R}^{+}$cells compared with that of normoxic treatment. In addition, $\mathrm{R}^{+}$cells exhibited higher levels of autophagy when compared with $\mathrm{R}^{-}$cells under normoxic and hypoxic conditions, which suggests that IGF-1R may promote autophagy.

Role of cell autophagy in ROS production and apoptosis under hypoxic conditions. ROS production and apoptosis were higher in $\mathrm{R}^{-}$cells compared with those in $\mathrm{R}^{+}$cells. Further investigation indicated that ROS production and apoptosis were increased after autophagy inhibition (3MA) treatment in all $\mathrm{R}^{+}$cells and $\mathrm{R}^{-}$cells compared with those without autophagy inhibition (3MA) treatment (Fig. 5). These suggested that autophagy may serve a protective role from ROS production and apoptosis.

Role of the PI3K/Akt/mTOR signaling pathway in $I G F-1 R$-induced autophagy under hypoxic conditions. As shown in Fig. 6, the $\mathrm{R}^{+}$cells displayed lower expression levels of PI3K-110 and mTOR, and higher expression of LC3-II under normoxic and hypoxic conditions relative to $\mathrm{R}^{-}$cells. Further study indicated that $\mathrm{p}$-AKT was decreased in $\mathrm{R}^{+}$cells compared with that of $\mathrm{R}^{-}$cells under normoxic conditions, however was increased significantly in $\mathrm{R}^{+}$cells following hypoxia treatment. When treated with the PI3K/Akt/mTOR inhibitor LY294002, $\mathrm{R}^{+}$and $\mathrm{R}^{-}$cells displayed lower levels of PI3K/p-Akt/p-mTOR and high LC3-II expression. These results indicated that the PI3K/Akt/mTOR signaling pathway may be involved in autophagy under normoxic and hypoxic conditions, which may be closely associated with IGF-1R.

\section{Discussion}

Hypoxia is a toxic factor that induces cell death through mitochondrial dysfunction, which is primarily caused by the production of ROS under hypoxic conditions $(23,24)$. Various pathological conditions, including myocardial I/R, stroke, cancer and irradiation, lead to tissue hypoxia, which alter 

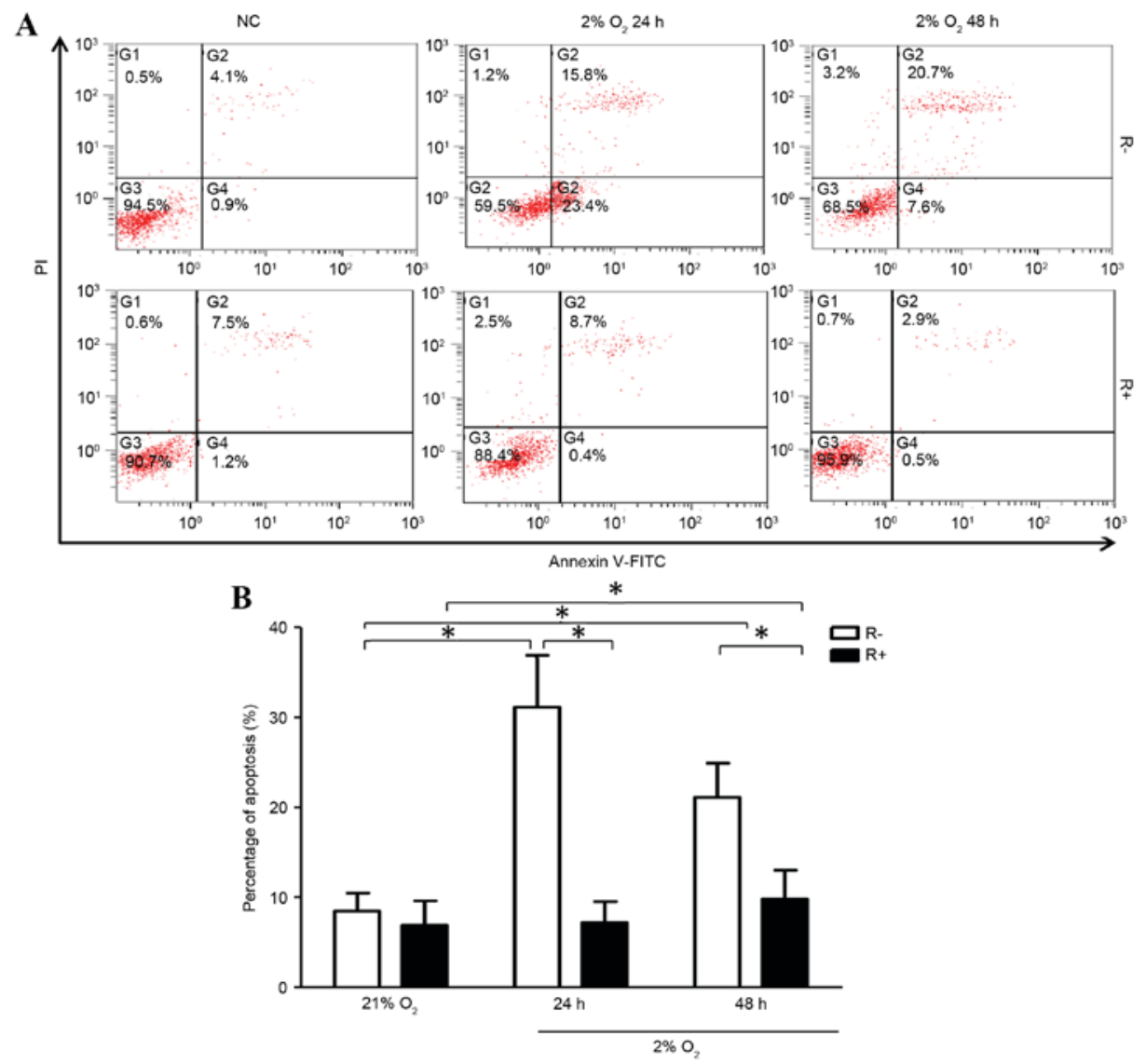

Figure 2. Effects of IGF-1R on hypoxia-induced apoptosis in $\mathrm{R}^{-}$and $\mathrm{R}^{+}$cells. (A) Representative pictures of apoptosis using flow cytometry analysis. (B) Quantification of the results apoptosis ratio. Data are presented as the mean \pm standard deviation of three independent experiments for each time point. Three independent images per time point were analyzed. "P $<0.05$. IGF-1R, insulin-like growth factor 1 receptor; PI, propidium iodide; FITC, fluorescein isothiocyanate; $\mathrm{R}^{+}$cells, mouse embryonic fibroblasts overexpressing IGF-1R; $\mathrm{R}^{-}$cells, mouse embryonic fibroblasts with targeted disruption of IGF-1R.
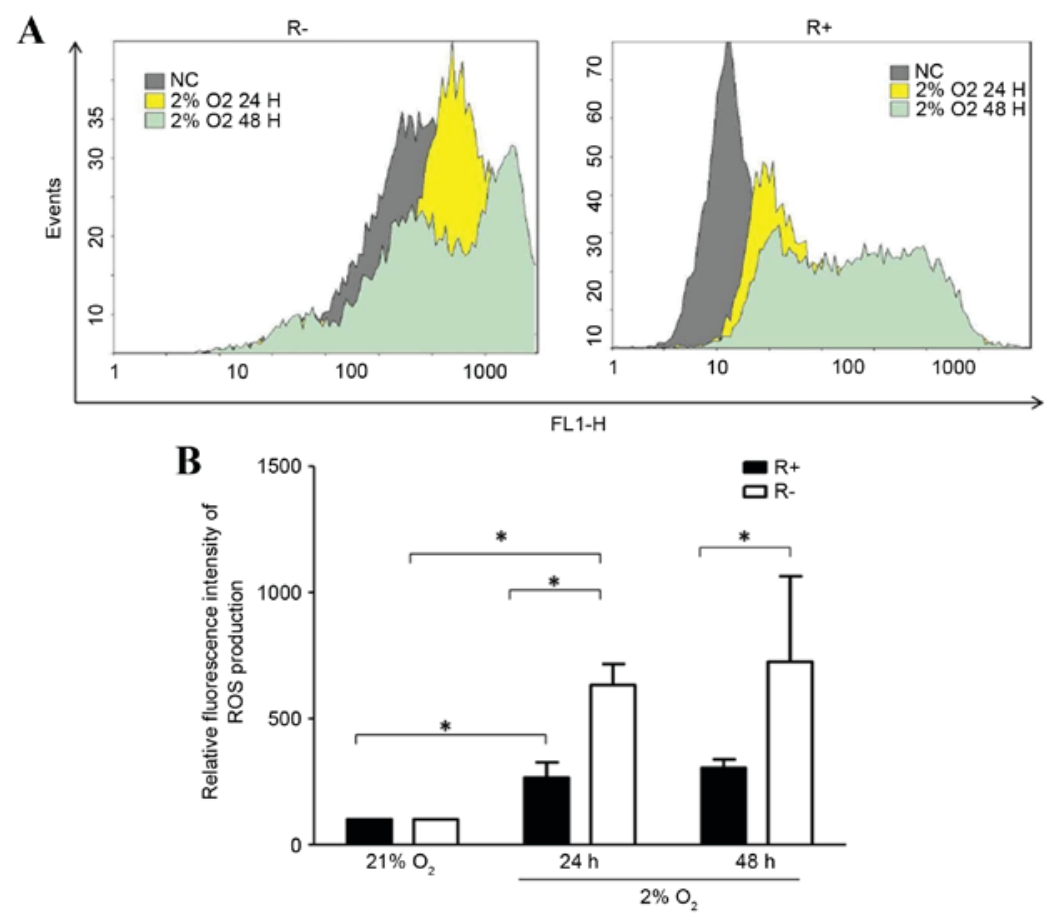

Figure 3. Effects of IGF-1R on hypoxia-induced ROS production in $\mathrm{R}^{-}$and $\mathrm{R}^{+}$cells. (A) The ratio of fluorescence intensity of ROS production has been shown (ROS production of $\mathrm{R}^{+}$cells under normoxic condition as the baseline). Cells were exposed to normoxic treatment $\left(21 \% \mathrm{O}_{2}, 24 \mathrm{~h}\right)$ or hypoxic treatment $(2 \%$ $\mathrm{O}_{2}, 24$ and 48 h). (B) Three independent experiments for each time point were analyzed. " $\mathrm{P}<0.05$. IGF-1R, insulin-like growth factor 1 receptor; ROS, reactive oxygen species; FL1-H, relative fluorescence intensity (height); NC, normoxic conditions; $\mathrm{R}^{+}$cells, mouse embryonic fibroblasts overexpressing IGF-1R; $\mathrm{R}^{\prime}$ cells, mouse embryonic fibroblasts with targeted disruption of IGF-1R. 
A
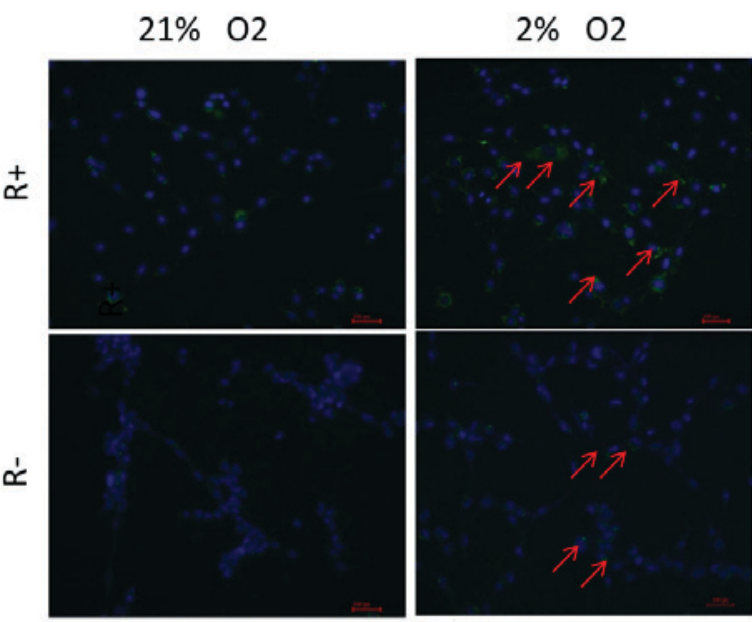

B

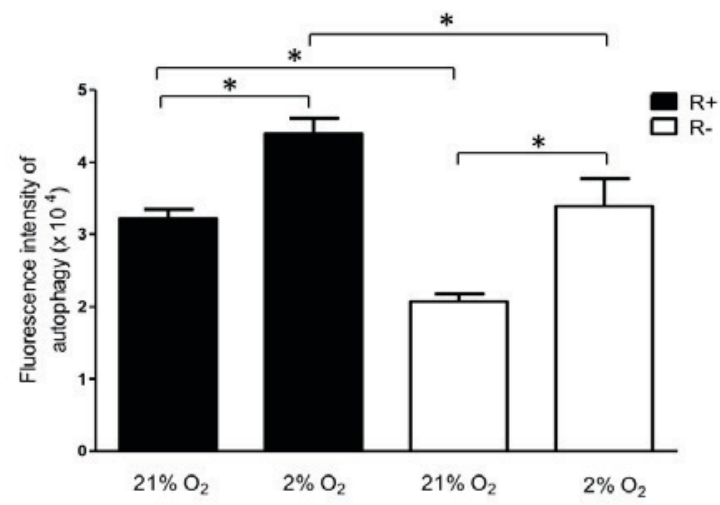

Figure 4. Effect of IGF-1R on hypoxia-induced autophagy in $\mathrm{R}^{-}$and $\mathrm{R}^{+}$cells (A) Representative fluorescence microscope images of autophagosomes, which were labeled with a green color. Magnification, x100; scale bar, $100 \mu \mathrm{m}$. (B) The rate of autophagosome formation was measured quantitatively under normoxic $\left(21 \% \mathrm{O}_{2}\right.$ for $\left.24 \mathrm{~h}\right)$ and hypoxic conditions $\left(2 \% \mathrm{O}_{2}\right.$ for $24 \mathrm{~h}$ ) using a fluorescence-intensity assay. A total of three independent images for each time point were analyzed. ${ }^{*} \mathrm{P}<0.05$ as indicated. IGF-1R, insulin-like growth factor 1 receptor; $\mathrm{R}^{+}$cells, mouse embryonic fibroblasts overexpressing IGF-1R; R- cells, mouse embryonic fibroblasts with targeted disruption of IGF-1R.

biological characteristics, including the production of ROS, autophagy and apoptosis. The results of the present study, demonstrated that low concentrations of oxygen induce ROS production and apoptosis in mouse embryonic fibroblasts. In addition, overexpression of IGF-1R may serve a protective role in cell death under hypoxic conditions through altering autophagy levels and ROS production.

The role of IGF-1R has received extensive attention due to its protective role in the neuronal system. It protects neural cells from loss and infarcted volume, and increases glial proliferation in the brain in a cerebral ischemia model (25-28). In addition, it serves a role in protecting against renal fibrosis-associated kidney disease, intestinal I/R injury and myocardial ischemia $(2,3,14)$. Autophagy is an intracellular lysosomal degradation process that maintains cellular homeostasis via the degradation and recycling of long-lived proteins and intracellular aggregates as well as damaged organelles, in order to generate small reusable molecules $(29,30)$. It is essential for the promotion of cell survival in stress conditions, such as starvation, oxidative
$\mathbf{A}$

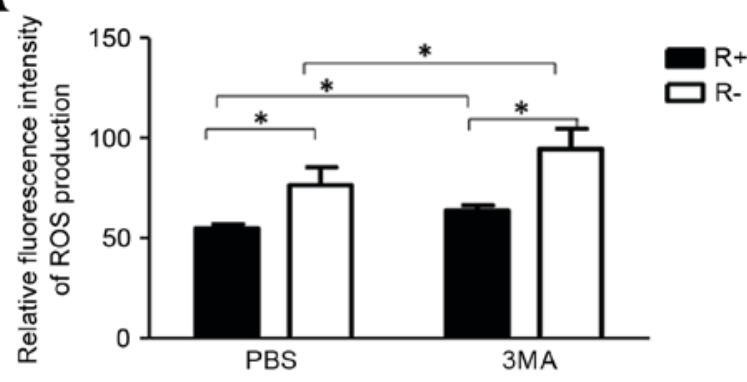

B

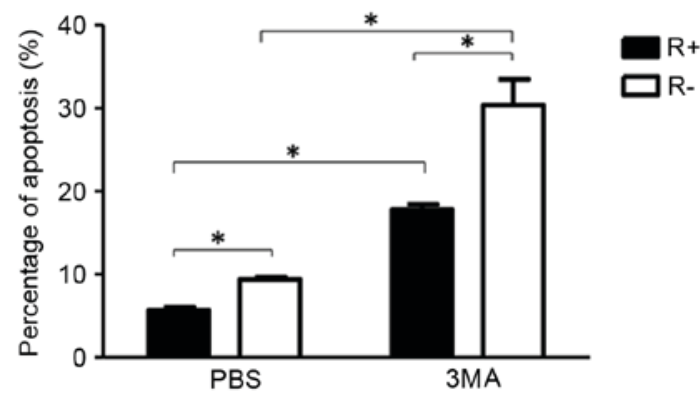

Figure 5. Effect of autophagy on ROS production and apoptosis under hypoxic conditions in $\mathrm{R}^{-}$and $\mathrm{R}^{+}$cells. (A) Cellular ROS levels during hypoxia was assessed using the H2DCFDA fluorescent probe in $\mathrm{R}^{-}$and $\mathrm{R}^{+}$cells following treatment with PBS or 3MA $(5 \mu \mathrm{M})$. (B) Apoptosis levels during hypoxia were assessed following treatment with PBS or 3MA $(5 \mu \mathrm{M}) .{ }^{*} \mathrm{P}<0.05$. ROS, reactive oxygen species; PBS, phosphate-buffered saline; 3MA, 3-methyladenine; $\mathrm{R}^{+}$cells, mouse embryonic fibroblasts overexpressing insulin-like growth factor 1 receptor; $\mathrm{R}^{-}$cells, mouse embryonic fibroblasts with targeted disruption of insulin-like growth factor 1 receptor.
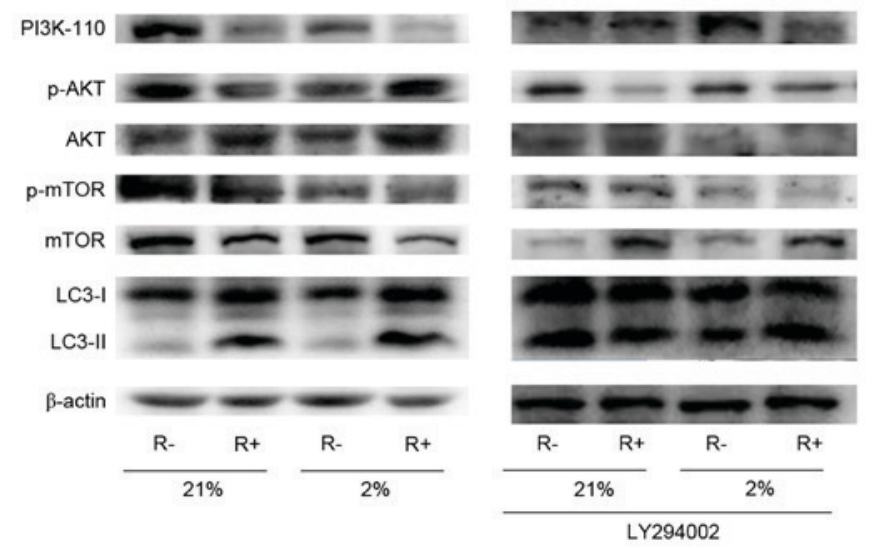

Figure 6. Association between IGF-1R and PI3K/Akt/mTOR signaling pathways and autophagy in $\mathrm{R}^{-}$and $\mathrm{R}^{+}$cells. The cells were cultured under normoxic $\left(21 \% \mathrm{O}_{2}\right)$ or hypoxic conditions $\left(2 \% \mathrm{O}_{2}\right)$ for $24 \mathrm{~h}$ with or without the PI3K inhibitor LY294002 $(10 \mu \mathrm{M})$. IGF-1R, insulin-like growth factor 1 receptor; PI3K, phosphatidylinositol 3-kinase; mTOR, mammalian target of rapamycin; p-, phosphorylated; Akt, threonine protein kinase B; LC3, autophagy marker light chain 3.

stress, and hypoxia; however, unregulated autophagy induces progressive consumption of cellular contents and results in autophagic cell death (31). Autophagy serves a protective role in cell survival in cells through the reduction of ROS. Inhibition of autophagy through chloroquine administration, or via prevention of K63 ubiquitination increases the formation of $\operatorname{ROS}(32,33)$. Hypoxia is a known inducer of 
autophagy (34). The present study demonstrated that the induction of autophagy and ROS production under hypoxic conditions, and autophagy inhibition by 3MA, led to a higher level of ROS production and cell apoptosis in $\mathrm{R}^{+}$and $\mathrm{R}^{-}$cells, which is in agreement with a previous study (35).

The signaling pathways involved in the progression of autophagy are complex, and the PI3K/Akt pathway has been widely studied $(36,37)$. The anti-apoptotic effects of IGF-1 are mediated by IGF-1R, together with the subsequent activation of $\mathrm{PI} 3 \mathrm{~K} / \mathrm{Akt} / \mathrm{mTOR}$ and other signaling pathways (38). However, the role of IGF-1 in autophagy remains unknown. An inhibitory effect of IGF-1 on autophagy has been observed in rat cardiomyocytes (39), human fibroblasts (40), and vascular cells from patients with atherosclerotic lesions (41). These effects are associated with rescuing mitochondrial metabolism and controlling the potentially harmful autophagic response. By contrast, IGF-1 promotes autophagy in H9c2 cell lines (42), HeLa cells (43) and Purkinje neurons (44). The depletion of IGF-1R inhibits mTORC2 and reduces the activation of protein kinase $\mathrm{C}$, which decreases the rate of clathrin-dependent endocytosis and impacts autophagosome-precursor formation (43). Furthermore, IGF-1 prevents the accumulation of autophagic vesicles and cell death by increasing the rate of autophagosome-to-lysosome fusion and degradation, thereby contributing to cell survival (44).

The current study investigated the effects of the IGF-1/PI3K/Akt/mTOR signaling pathway in the progression of autophagy in mouse embryonic fibroblasts. The results indicated that IGF-1R-overexpressing cells exhibit lower expression of PI3K-110, p-Akt, p-mTOR/mTOR and higher expression levels of LC3-II under normoxic and hypoxic conditions. When treated with the PI3K/Akt/mTOR inhibitor LY294002, these cell types exhibited lower PI3K/Akt/mTOR signaling activation and higher autophagy levels, which indicated that IGF-1R-induced cell survival during hypoxia, which may be dependent on autophagy initiation by suppressing the $\mathrm{PI} 3 \mathrm{~K} / \mathrm{Akt} / \mathrm{mTOR}$-signaling pathway.

The present study demonstrated that overexpression of IGF-1R is correlated with reduced ROS production, increased autophagy and cell viability under hypoxic conditions. It is possible that, binding of IGF-1 to IGF-1R suppressed the $\mathrm{PI} 3 \mathrm{~K} / \mathrm{Akt} / \mathrm{mTOR}$ signaling pathway, which promoted autophagy and scavenging of redundant cellular ROS during hypoxia. These results reveal novel mechanisms for IGF-1R-associated cell survival, which may be good to know I/R- and H/I associated hypoxia-agravated normal tissue injury well.

\section{Acknowledgements}

The current study was supported by the Scientific Research Foundation for the Returned Overseas Chinese Scholars from the China State Education Ministry (No. N130204), the National Natural Science Foundation of China (Nos. 81202148 and 31370838) and the Shanghai Pujiang Program (No. 13P1401600).

\section{References}

1. Liu W, D'Ercole JA and Ye P: Blunting type 1 insulin-like growth factor receptor expression exacerbates neuronal apoptosis following hypoxic/ischemic injury. BMC Neurosci 12: 64, 2011.
2. Du L, Yu Y, Ma H, Lu X, Ma L, Jin Y and Zhang H: Hypoxia enhances protective effect of placental-derived mesenchymal stem cells on damaged intestinal epithelial cells by promoting secretion of insulin-like growth factor-1. Int J Mol Sci 15: 1983-2002, 2014

3. Li HX, Zhou YF, Zhao X, Jiang B and Yang XJ: GATA-4 protects against hypoxia-induced cardiomyocyte injury: Effects on mitochondrial membrane potential. Can J Physiol Pharmacol 92: 669-678, 2014.

4. Caldwell CC, Tschoep J and Lentsch AB: Lymphocyte function during hepatic ischemia/reperfusion injury. J Leukoc Biol 82: 457-464, 2007.

5. Ildefonso JA and Arias-Diaz J: Pathophysiology of liver ischemia-reperfusion injury. Cir Esp 87: 202-209, 2010 (In Spanish).

6. Vujaskovic Z, Anscher MS, Feng QF, Rabbani ZN, Amin K Samulski TS, Dewhirst MW and Haroon ZA: Radiation-induced hypoxia may perpetuate late normal tissue injury. Int J Radiat Oncol Biol Phys 50: 851-855, 2001.

7. Vujaskovic Z, Marks LB and Anscher MS: The physical parameters and molecular events associated with radiation-induced lung toxicity. Semin Radiat Oncol 10: 296-307, 2000.

8. Luo Y, Liu X, Zheng Q, Wan X, Ouyang S, Yin Y, Sui X, Liu J and Yang $\mathrm{X}$ : Hydrogen sulfide prevents hypoxia-induced apoptosis via inhibition of an $\mathrm{H} 2 \mathrm{O} 2$-activated calcium signaling pathway in mouse hippocampal neurons. Biochem Biophys Res Commun 425: 473-477, 2012.

9. Kim BM and Chung HW: Hypoxia/reoxygenation induces apoptosis through a ROS-mediated caspase-8/Bid/Bax pathway in human lymphocytes. Biochem Biophys Res Commun 363: 745-750, 2007.

10. Peng C, Rao W, Zhang L, Wang K, Hui H, Wang L, Su N, Luo P, Hao YL, Tu Y, et al: Mitofusin 2 ameliorates hypoxia-induced apoptosis via mitochondrial function and signaling pathways. Int J Biochem Cell Biol 69: 29-40, 2015.

11. Kuo CY, Chiu YC, Lee AY and Hwang TL: Mitochondrial Lon protease controls ROS-dependent apoptosis in cardiomyocyte under hypoxia. Mitochondrion 23: 7-16, 2015.

12. Laron Z: Insulin-like growth factor 1 (IGF-1): A growth hormone. Mol Pathol 54: 311-316, 2001

13. Chisalita SI and Arnqvist HJ: Insulin-like growth factor I receptors are more abundant than insulin receptors in human micro- and macrovascular endothelial cells. Am J Physiol Endocrinol Metab 286: E896-E901, 2004.

14. Liang M, Woodard LE, Liang A, Luo J, Wilson MH, Mitch WE and Cheng J: Protective role of insulin-like growth factor-1 receptor in endothelial cells against unilateral ureteral obstruction-induced renal fibrosis. Am J Pathol 185: 1234-1250, 2015.

15. Custodio RJ, do Carmo Custodio VI, Scrideli CA, Sader Milani SL, Cervi MC, Cupo P and Martinelli CE Jr: Impact of hypoxia on IGF-I, IGF-II, IGFBP-3, ALS and IGFBP-1 regulation and on IGF1R gene expression in children. Growth Horm IGF Res 22: 186-191, 2012.

16. Morgan BL and Chao CR: The effects of hypoxia on growth cones in the ovine fetal brain. J Matern Fetal Neonatal Med 16: 55-59, 2004.

17. Heck S, Lezoualc'h F, Engert $S$ and Behl C: Insulin-like growth factor-1-mediated neuroprotection against oxidative stress is associated with activation of nuclear factor kappaB. J Biol Chem 274: 9828-9835, 1999.

18. Baregamian N, Song J, Jeschke MG, Evers BM and Chung DH: IGF-1 protects intestinal epithelial cells from oxidative stress-induced apoptosis. J Surg Res 136: 31-37, 2006.

19. Maldonado C, Cea P, Adasme T, Collao A, Díaz-Araya G, Chiong $\mathrm{M}$ and Lavandero S: IGF-1 protects cardiac myocytes from hyperosmotic stress-induced apoptosis via CREB. Biochem Biophys Res Commun 336: 1112-1118, 2005.

20. Yu D, Watanabe H, Shibuya $\mathrm{H}$ and Miura M: Redundancy of radioresistant signaling pathways originating from insulin-like growth factor I receptor. J Biol Chem 278: 6702-6709, 2003.

21. Yu D, Shibuya $\mathrm{H}$ and Miura M: Roles of the insulin-like growth factor I receptor C-terminus in cellular radioresistance. Biochem Biophys Res Commun 311: 174-178, 2003.

22. Livak KJ and Schmittgen TD: Analysis of relative gene expression data using real-time quantitative PCR and the 2(-Delta Delta C(T)) Method. Methods 25: 402-408, 2001.

23. Martin M, Lefaix J and Delanian S: TGF-betal and radiation fibrosis: A master switch and a specific therapeutic target? Int J Radiat Oncol Biol Phys 47: 277-290, 2000.

24. Murphy MP: How mitochondria produce reactive oxygen species. Biochem J 417: 1-13, 2009. 
25. Lopez-Lopez C, LeRoith D and Torres-Aleman I: Insulin-like growth factor I is required for vessel remodeling in the adult brain. Proc Natl Acad Sci USA 101: 9833-9838, 2004.

26. Conti E, Carrozza C, Capoluongo E, Volpe M, Crea F, Zuppi C and Andreotti F: Insulin-like growth factor-1 as a vascular protective factor. Circulation 110: 2260-2265, 2004.

27. Adams MM, Elizabeth Forbes M, Constance Linville M, Riddle DR, Sonntag WE and Brunso-Bechtold JK: Stability of local brain levels of insulin-like growth factor-I in two well-characterized models of decreased plasma IGF-I. Growth Factors 27: 181-188, 2009.

28. Carro E, Spuch C, Trejo JL, Antequera D and Torres-Aleman I: Choroid plexus megalin is involved in neuroprotection by serum insulin-like growth factor I. J Neurosci 25: 10884-10893, 2005.

29. Avivar-valderas A, Bobrovnikova-Marjon E, Alan Diehl J, Bardeesy N, Debnath J and Aguirre-Ghiso JA: Regulation of autophagy during ECM detachment is linked to a selective inhibition of mTORC1 by PERK. Oncogene 32: 4932-4940, 2013.

30. Liu B, Wen X and Cheng Y: Survival or death: Disequilibrating the oncogenic and tumor suppressive autophagy in cancer. Cell Death Dis 4: e892, 2013.

31. Eisenberg-Lerner A, Bialik S, Simon HU and Kimchi A: Life and death partners: Apoptosis, autophagy and the cross-talk between them. Cell Death Differ 16: 966-975, 2009.

32. Rouschop KM, Ramaekers CH, Schaaf MB, Keulers TG, Savelkouls KG, Lambin P, Koritzinsky M and Wouters BG: Autophagy is required during cycling hypoxia to lower production of reactive oxygen species. Radiother Oncol 92: 411-416, 2009.

33. Farombi EO: Genotoxicity of chloroquine in rat liver cells: Protective role of free radical scavengers. Cell Biol Toxicol 22: 159-167, 2006

34. Sun Y, Xing X, Liu Q, Wang Z, Xin Y, Zhang P, Hu C and Liu Y: Hypoxia-induced autophagy reduces radiosensitivity by the HIF-1 $\alpha / \mathrm{miR}-210 / \mathrm{Bcl}-2$ pathway in colon cancer cells. Int J Oncol 46: 750-756, 2015.

35. Liu Q, Sun Y, Lv Y, Le Z, Xin Y, Zhang P and Liu Y: TERT alleviates irradiation-induced late rectal injury by reducing hypoxia-induced ROS levels through the activation of NF- $\kappa \mathrm{B}$ and autophagy. Int J Mol Med 38: 785-793, 2016.
36. Shao X, Lai D, Zhang L and Xu H: Induction of autophagy and apoptosis via PI3K/AKT/TOR pathways by azadirachtin a in spodoptera litura cells. Sci Rep 6: 35482, 2016.

37. Fan XJ, Wang Y, Wang L and Zhu M: Salidroside induces apoptosis and autophagy in human colorectal cancer cells through inhibition of PI3K/Akt/mTOR pathway. Oncol Rep 36: 3559-3567, 2016.

38. Saetrum Opgaard O and Wang PH: IGF-I is a matter of heart. Growth Horm IGF Res 15: 89-94, 2005.

39. Troncoso R, Vicencio JM, Parra V, Nemchenko A, Kawashima Y, Del Campo A, Toro B, Battiprolu PK, Aranguiz P, Chiong M, et al: Energy-preserving effects of IGF-1 antagonize starvation-induced cardiac autophagy. Cardiovasc Res 93: 320-329, 2012.

40. Bitto A, Lerner C, Torres C, Roell M, Malaguti M, Perez V, Lorenzini A, Hrelia S, Ikeno Y, Matzko ME, et al: Long-term IGF-I exposure decreases autophagy and cell viability. PLoS One 5: e12592, 2010

41. Jia G, Cheng G, Gangahar DM and Agrawal DK: Insulin-like growth factor-1 and TNF-alpha regulate autophagy through c-jun N-terminal kinase and Akt pathways in human atherosclerotic vascular smooth cells. Immunol Cell Biol 84: 448-454, 2006.

42. Aki T, Yamaguchi K, Fujimiya $\mathrm{T}$ and Mizukami Y: Phosphoinositide 3-kinase accelerates autophagic cell death during glucose deprivation in the rat cardiomyocyte-derived cell line H9c2. Oncogene 22: 8529-8535, 2003.

43. Renna M, Bento CF, Fleming A, Menzies FM, Siddiqi FH, Ravikumar B, Puri M, Garcia-Arencibia M, Sadiq O, Corrochano S, et al: IGF-1 receptor antagonism inhibits autophagy. Hum Mol Genet 22: 4528-4544, 2013.

44. Bains M, Florez-McClure ML and Heidenreich KA: Insulin-like growth factor-I prevents the accumulation of autophagic vesicles and cell death in Purkinje neurons by increasing the rate of autophagosome-to-lysosome fusion and degradation. J Biol Chem 284: 20398-20407, 2009. 